\title{
Análise do processo de produção e compreensão do prontuário de enfermagem no ambiente hospitalar
}

Analysis of the production process and understanding of the medical record in the hospital environment

Análisis del proceso de producción y comparación del prontuário de enfermagem no ambiente hospitalar

Thalia Jeovana da Silva Pereira ORCID: https://orcid.org/0000-0003-0323-5318 E-mail:thaliajeovana96@gmail.com

Wenderson Costa da Silva ORCID: https://orcid.org/0000-0001-6031-9775

Universidade Estadual do Maranhão, Brasil

E-mail: wendersoncosta09@hotmail.com

Hálmisson D'Árley Santos Siqueira

ORCID: https://orcid.org/0000-0001-9831-5892

Centro Universitário de Ciências e Tecnologia do Maranhão, Brasil E-mail: halmisson@yahoo.com.br

Rogério Cruz Mendes

ORCID: https://orcid.org/0000-0002-9339-6133

Centro Universitário de Ciências e Tecnologia do Maranhão, Brasil E-mail: rogeriocruz82@yahoo.com

Marta Valeria Soares Chaves

ORCID: https://orcid.org/0000-0002-3797-3696

Centro Universitário de Ciências e Tecnologia do Maranhão, Brasil E-mail: martavaleria706@gmail.com

Brunna Matos Sousa

ORCID: https://orcid.org/0000-0002-7009-6488 Centro Universitário de Ciências e Tecnologia do Maranhão, Brasil E-mail: brunnamattos4@gmail.com

Railson Muniz de Sousa

ORCID: https://orcid.org/0000-0003-2257-2061

Centro Universitário de Ciências e Tecnologia do Maranhão, Brasil E-mail: raylsonmuniz007@gmail.com

Karen Laís Azevêdo Oliveira Sousa

ORCID: https://orcid.org/0000-0002-0308-417X Centro Universitário de Ciências e Tecnologia do Maranhão, Brasil E-mail: Karen.lais05@gmail.com

Lisianne Natália Santos Silva

ORCID: https://orcid.org/0000-0002-2574-399X

Centro Universitário de Ciências e Tecnologia do Maranhão, Brasil E-mail: lisyanenathalia6@gmail.com

Tatila Shinaira Soares Chaves

ORCID: https://orcid.org/0000-0002-1443-9773

Centro Universitário de Ciências e Tecnologia do Maranhão, Brasil E-mail: tatylachaves@gmail.com

Linccon Fricks Hernandes

ORCID: https://orcid.org/0000-0002-7642-3080

Escola Superior de Ciências da Santa Casa de Misericórdia de Vitória, Brasil

E-mail: fricksjr@hotmail.com

Maria Gabrielle Sobral da Silva

ORCID: https://orcid.org/0000-0002-7493-3060

Centro Universitário de Ciências e Tecnologia do Maranhão, Brasil

E-mail: mariagabrielle980@gmail.com

Melissa Torres Soares Rabêlo

ORCID: https://orcid.org/0000-0002-5344-9894

Centro Universitário de Ciências e Tecnologia do Maranhão, Brasil

E-mail: melissatdss11@gmail.com 


\author{
Layse Siqueira Costa Miranda \\ ORCID: https://orcid.org/0000-0001-5942-4666 \\ Centro Universitário de Ciências e Tecnologia do Maranhão, Brasil \\ E-mail: layse62@gmail.com
}

\begin{abstract}
Resumo
O presente estudo objetivou analisar por meio da literatura, as evidências científicas que envolvem os processos de produção e compreensão do prontuário de enfermagem no ambiente hospitalar. Trata-se de uma revisão integrativa de literatura, em que formulou-se a seguinte questão: "Quais as evidências cientificas sobre o processo de produção e compreensão do prontuário de enfermagem no ambiente hospitalar?". Montou-se uma estratégia PICo, na qual por meio de descritores e palavras-chave foram consultadas as bases de dados da National Library of Medicine; BVS (Biblioteca Virtual da Saúde), e CINAHL (Cumulative Index to Nursing and Allied Health Literature). Foram incluídos treze estudos nesta revisão. A maioria dos estudos versou sobre os componentes chaves para a elaboração do prontuário, enfatizando sua finalidade, qualidade e a utilização no ambiente hospitalar; as principais conformidades e não conformidades; as implicações segundo as normas e regulamentos; e as dificuldades encontradas pelos profissionais referentes ao setor e a fase da assistência. Com relação às falhas nos registros de enfermagem, estudos citaram que a maioria dos profissionais desconhece os requisitos da instituição, as legislações vigentes e o código de ética. Conclui-se que os profissionais de enfermagem devem adotar medidas de capacitação permanente para a adesão correta do prontuário, buscar resolutividades sobre o serviço fragmentado, e aprender com seus próprios erros, para que não haja complicações nas ações a serem realizadas no que tange ao paciente hospitalizado.
\end{abstract}

Palavras-chave: Profissionais de enfermagem; Registros de enfermagem; Hospitais.

\begin{abstract}
This study aimed to analyze, through the literature, the scientific evidence that involves the processes of production and understanding of the nursing record in the hospital environment. It is an integrative literature review, in which the following question was asked: "What is the scientific evidence about the process of production and understanding of the nursing record in the hospital environment?". A PICo strategy was set up, in which, through descriptors and keywords, the databases of the National Library of Medicine were consulted; VHL (Virtual Health Library), and CINAHL (Cumulative Index to Nursing and Allied Health Literature). Thirteen studies were included in this review. Most studies dealt with the key components for the elaboration of the medical record, emphasizing its purpose, quality and use in the hospital environment; the main conformities and non-conformities; the implications under rules and regulations; and the difficulties encountered by professionals regarding the sector and the assistance phase. Regarding the flaws in the nursing records, studies cited that most professionals are unaware of the institution's requirements, the current legislation and the code of ethics. It is concluded that nursing professionals must adopt permanent training measures for correct adherence to medical records, seek resolution on fragmented service, and learn from their own mistakes, so that there are no complications in the actions to be performed with regard to the patient hospitalized.
\end{abstract}

Keywords: Nursing professionals; Nursing records; Hospitals.

\title{
Resumen
}

Este estudio tuvo como objetivo analizar, a través de la literatura, la evidencia científica que involucra los procesos de producción y comprensión del registro de enfermería en el ámbito hospitalario. Se trata de una revisión integradora de la literatura, en la que se planteó la siguiente pregunta: “¿Cuál es la evidencia científica sobre el proceso de producción y comprensión del registro de enfermería en el ámbito hospitalario?”. Se estableció una estrategia PICo, en la cual, a través de descriptores y palabras clave, se consultaron las bases de datos de la Biblioteca Nacional de Medicina; BVS (Biblioteca Virtual en Salud) y CINAHL (Índice Acumulativo de Literatura de Enfermería y Afines en Salud). Se incluyeron trece estudios en esta revisión. La mayoría de los estudios abordaron los componentes clave para la elaboración de la historia clínica, destacando su finalidad, calidad y uso en el ámbito hospitalario; las principales conformidades y no conformidades; las implicaciones bajo las reglas y regulaciones; y las dificultades encontradas por los profesionales en el sector y la fase asistencial. En cuanto a las fallas en los registros de enfermería, estudios citan que la mayoría de los profesionales desconocen los requisitos de la institución, la legislación vigente y el código ético. Se concluye que los profesionales de enfermería deben adoptar medidas de formación permanente para la correcta adherencia a la historia clínica, buscar la resolución del servicio fragmentado y aprender de sus propios errores, para que no existan complicaciones en las acciones a realizar con respecto al paciente hospitalizado.

Palabras clave: Profesionales de enfermería; Registros de enfermería; Hospitales. 


\section{Introdução}

O termo prontuário origina-se do latim "Promptuarium" que significa um lugar e/ou deposito, onde são guardados dados importantes, podendo ser consultado a qualquer momento que precisar. $\mathrm{O}$ acervo documental é um instrumento de atuação da equipe de enfermagem, um conjunto de documentos fidedigno que retratam a realidade do paciente e a assistência prestada, o mesmo deve ser produzido e armazenado adequadamente. Sobretudo, o prontuário consiste em aspecto ético-legal e demandas jurídicas, as informações fornecidas nele usufruem de presunção de veracidade. Caso o cliente não esteja satisfeito com as informações registradas no prontuário, caberá o mesmo e ao profissional o ônus de confirmar a ausência de verdade ou a inexatidão que está anexada no prontuário (Coltri \& Silva, 2019).

Os provedores de cuidado hospitalar são os que se mantém próximo do paciente durante as 24 horas, coletando os dados que abordam à prática de enfermagem, quer para conduzir os cuidados, quer para distribuir com os diversos profissionais. A comunicação, entretanto, é o componente imprescindível na assistência de enfermagem. Os registros de enfermagem, a forma escrita da comunicação particularmente no prontuário do cliente, são indispensáveis para a continuidade do cuidado. Contudo os registros de enfermagem as vezes não se constituem em motivo de preocupação entre os integrantes da equipe, que os produzem de maneira rápida e sem clareza (Candido, Cunha, \& Munhoz, 2018).

As anotações da equipe de enfermagem referente a prática do cuidado, são executadas para se ter um meio de comunicação permanente entre os diferentes profissionais de saúde, concedendo a equipe transmitir, receber e acessar as informações no prontuário, em que o mesmo resultará aperfeiçoar a qualidade e continuidade da assistência, e ao seu planejamento. É de suma importância a efetuação das evoluções, prescrições, registros e anotações de enfermagem redigidas de maneira correta sobre o estado de saúde do cliente (Santana \& Araújo, 2016).

Várias condições podem interferir na padronização, na qualidade das anotações e no progresso do trabalho, dentre eles apontam: omitir ou distorcer informações relativas ao cuidado individual; uso de abreviaturas não padronizadas, demasia de clientes sob a obrigação da equipe de enfermagem no ambiente do cuidado; cuidadores sobrecarregados por tarefas de estrutura gerencial; dificuldade para a elaboração e interpretação das anotações; inclusão de documentações sem a devida habilitação profissional da equipe; desinformações das situações éticas e legais dos registros; e a ausência de organização correta e equipes bem preparadas e direcionadas (Araujo, Diniz, \& Silva, 2017).

A qualidade da assistência é uma busca constante dos serviços hospitalares, contudo, os profissionais de enfermagem precisam ser orientados e qualificados para produzir os registros e anotações de enfermagem, para identificar e corrigir os principais erros durante o processo, para que fique conciso a importância e a correta forma do preenchimento no prontuário do paciente as condutas executadas e o cuidado empregado desde a internação até a alta hospitalar, apenas desta forma poderemos alcançar a melhoraria da qualidade da assistência e reduzir as intercorrências nas instituições hospitalares. Os profissionais de saúde precisam conhecer e se apoderar das legislações vigentes quanto à maneira correta e a valorização dos registros para respaldo das atuações realizadas (Camargo \& Pereira, 2017).

Desse modo, o profissional enfermeiro inegavelmente, rebaixa o seu serviço, dá prejuízo na prática e em todo conhecimento adquirido quando não registra corretamente as informações fornecidas pelo cliente e a família, a inexistência destes evidenciam a falta de embasamento na sua escolha de decisão, de planejamento da assistência e direcionamento das ações dos profissionais de enfermagem, inclusive, também aponta a construção do prontuário e a depreciação da avaliação dos resultados atingidos para o feedback do sistema de melhoria da qualidade do exercício profissional (Candido et al., 2018).

Por fim, este estudo justifica-se pela necessidade de sedimentar o prontuário como um documento único e resguardo de natureza sigilosa, pesquisar as grandes intercorrências contidas no prontuário, abordando a importância de analisar como os provedores de saúde conseguem desenvolver uma assistência integral, referente ao impacto da desorganização de prontuários, 
desvalorização dos dados, barreiras no processo de comunicação, a má qualidade de preenchimentos das informações, variações na prática dos registros e dificuldades no processo de continuidade no tratamento do paciente.

Este estudo buscou discernir as possíveis falhas existentes no processo de anotações registradas no prontuário, enfatizando assim, as principais implicações não só enfrentadas pelos pacientes, mas também, aos provedores de saúde a respeito do tratamento e o comprometimento da assistência.

Diante dessa questão, a caracterização do estudo se assenta pela constante necessidade de conhecimentos dos profissionais de enfermagem sobre esta condição, para que possam apreciar a importância sobre a valorização crucial da qualidade das anotações e investirem na excelência do seu trabalho, que muitas das vezes passam despercebidos pela equipe que atua, apesar de todo o amparo teórico-cientifico, encontram-se em carência para produzir e lidar com os registros de enfermagem, ocorrendo assim, a desvalorizações dos dados, e comprometendo a seguridade do paciente, contudo, os registros deve-se satisfazer os requisitos necessários, com preenchimento efetivo, de modo a qualificar as ações de promoção, cura e reabilitação da saúde do paciente.

Com base no exposto, traçou-se a seguinte problemática: quais as evidências cientificas sobre o processo de produção e compreensão do prontuário de enfermagem no ambiente hospitalar?

O objetivo geral dessa pesquisa foi analisar, por meio da literatura, as evidências científicas que envolvem os processos de produção e compreensão do prontuário de enfermagem no ambiente hospitalar. Especificamente objetivou-se: identificar os autores dos registros presentes nos prontuários que mais ocasionam erros; determinar o setor e a fase da assistência em que ocorrem os maiores números de erros identificados nos prontuários; avaliar conformidades e não conformidades das informações obtidas nos registros de enfermagem; e destacar as características dos registros de enfermagem requeridos nos prontuários dos pacientes hospitalizados.

\section{Metodologia}

O estudo trata-se de uma pesquisa bibliográfica do tipo revisão integrativa da literatura. Este método foi escolhido por possibilitar uma síntese e análise mais aprofundada do conhecimento científico que já foram produzidos sobre o tema "análise do processo de produção e compreensão do prontuario de emfermagem no ambiente hospitalar: uma revisão integrativa". Esta revisão utilizou-se a metodologia proposta no estudo de oliveira et al. (2016).

O tema determinou a construção da estratégia PICo, que representa um acrônimo para Paciente ou Problema (P), Interesse (I), Contexto (Co), na qual foi utilizada para a geração da questão norteadora desta revisão integrativa da literatura: "Quais as evidências cientificas sobre o processo de produção e compreensão do prontuário de enfermagem no ambiente hospitalar?".

Para a localização dos estudos relevantes, que respondessem à pergunta de pesquisa, utilizou-se de descritores indexados e não indexados (palavras-chave) nos idiomas português, inglês e espanhol. Os descritores foram obtidos a partir do Medical Subject Headings (MESH), dos Descritores em Ciências da Saúde (DeCS) e dos títulos da Cumulative Index to Nursing and Allied Health Literature (CINAHL), como mostra o Quadro 1.

Consultaram-se por meio de descritores e palavras-chave as bases de dados PubMed da National Library of Medicine; Biblioteca Virtual da Saúde (BVS), coordenada pela BIREME e composta de bases de dados bibliográficas produzidas pela Rede BVS, como a Literatura Latino-Americana e do Caribe em Ciências da Saúde (LILACS), além da base de dados MEDLINE (Medical Literature Analysis and Retrieval System Online) e outros tipos de fontes de informação; e CINAHL. 
Quadro 1. Elementos da estratégia PICo, descritores e palavras-chave utilizados. Caxias, MA, Brasil, 2020.

\begin{tabular}{|c|c|c|c|c|c|}
\hline & ELEMENTOS & MESH & DECS & CINAHL & $\begin{array}{l}\text { PALAVRA- } \\
\text { CHAVE }\end{array}$ \\
\hline $\mathbf{P}$ & $\begin{array}{l}\text { "Profissionais de } \\
\text { enfermagem" }\end{array}$ & $\begin{array}{c}\text { "Nurse } \\
\text { Practitioners" }\end{array}$ & $\begin{array}{c}\text { "Nurse } \\
\text { Practitioners" } \\
\text { "Enfermeras } \\
\text { Practicantes" } \\
\text { "Profissionais de } \\
\text { enfermagem" }\end{array}$ & $\begin{array}{c}\text { "Nurse } \\
\text { Practitioners" }\end{array}$ & $\begin{array}{c}\text { "Nurse } \\
\text { Practitioners" } \\
\text { "Enfermeras } \\
\text { Practicantes" } \\
\text { "Profissionais de } \\
\text { enfermagem" }\end{array}$ \\
\hline I & $\begin{array}{l}\text { "Registros de } \\
\text { enfermagem" }\end{array}$ & $\begin{array}{l}\text { "Nursing } \\
\text { Records" }\end{array}$ & $\begin{array}{l}\text { "Nursing Records" } \\
\text { "Registros de } \\
\text { enfermería" } \\
\text { "Registros de } \\
\text { enfermagem" }\end{array}$ & $\begin{array}{l}\text { "Nursing } \\
\text { Records" }\end{array}$ & $\begin{array}{l}\text { "Nursing Records" } \\
\text { "Registros de } \\
\text { enfermería" } \\
\text { "Registros de } \\
\text { enfermagem" }\end{array}$ \\
\hline Co & "Hospitais" & "Hospitals" & $\begin{array}{l}\text { "Hospitals" } \\
\text { "Hospitales" } \\
\text { "Hospitais" }\end{array}$ & "Hospitals" & $\begin{array}{l}\text { "Hospitals" } \\
\text { "Hospitales" } \\
\text { "Hospitais" }\end{array}$ \\
\hline
\end{tabular}

Fonte: Descritores, Títulos e Palavras-chaves (2020).

Os termos utilizados durante a pesquisa foram classificados e combinados nos bancos de dados, resultando em estratégias específicas de cada base, conforme o Quadro 2.

Quadro 2. Estratégias de busca utilizadas nas bases de dados BIREME, PUBMED e CINAHL. Caxias, MA, Brasil, 2020.

\begin{tabular}{|c|c|c|c|c|}
\hline $\begin{array}{l}\text { BASE DE } \\
\text { DADOS }\end{array}$ & ESTRATÉGIA DE BUSCA & 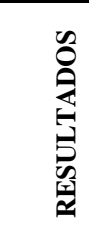 & 冚 & 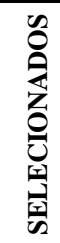 \\
\hline $\begin{array}{l}\text { BIREME } \\
\text { (descritores } \\
\text { Decs) }\end{array}$ & $\begin{array}{l}\text { tw:((tw:(profissionais de enfermagem })) \text { AND (tw:(registros de } \\
\text { enfermagem })) \text { AND (tw:(hospital })))(\text { instance:"regional") }\end{array}$ & 429 & 90 & 8 \\
\hline $\begin{array}{c}\text { PUBMED } \\
\text { (descriptors } \\
\text { MeSH) }\end{array}$ & $\begin{array}{l}\text { ("nurse practitioners"[MeSH Terms] OR ("nurse"[All Fields] } \\
\text { AND "practitioners"[All Fields]) OR "nurse } \\
\text { practitioners"[All Fields]) AND ("nursing records"[MeSH } \\
\text { Terms] OR ("nursing"[All Fields] AND "records"[All Fields]) } \\
\text { OR "nursing records"[All Fields]) } \\
\end{array}$ & 388 & 50 & 3 \\
\hline $\begin{array}{l}\text { CINAHL } \\
\text { (CINAHL } \\
\text { Headings) }\end{array}$ & Nurse Practitioners AND Nursing Records & 60 & 4 & 2 \\
\hline
\end{tabular}

Fonte: Bases de dados (2020).

Como critérios de inclusão utilizaram-se estudos disponíveis em sua totalidade, publicados nos últimos seis anos, de 2015 até 2020, nos idiomas Português, Espanhol e Inglês. Foram excluídos da busca inicial capítulos de livros, resumos, textos incompletos, teses de doutorado, dissertações de mestrados, monografias, relatos técnicos e outras formas de publicação que 
não fossem artigos científicos completos, foram incluídos ao estudo artigos científicos que respondesse a questão norteadora, diretrizes e protocolos em relação ao problema.

A análise para seleção dos estudos foi realizada em duas fases. Na primeira, os estudos foram pré-selecionados de acordo com os critérios de inclusão e exclusão, e conforme a estratégia de funcionamento e busca de cada base de dados.

Foram encontrados quatrocentos e vinte e nove (429) estudos como busca geral na base de dados da BIREME, sendo que limitando a busca utilizando os filtros, para artigos com texto completo realizado com humanos e nos últimos seis anos, obteve-se noventa (90) estudos, após a leitura dos títulos e resumos foram selecionados apenas oito (8) estudos o qual respondiam à questão norteadora da pesquisa, estando todos no idioma português.

$\mathrm{Na}$ base de dados PUBMED, o descritor Contexto (Co) não foi utilizado para se ter maior abrangência de estudos. Com a busca adaptada foram encontrados trezentos e oitenta e oito (388) estudos no geral, quando aplicado na pesquisa os filtros, que limitam-se por texto completo dos últimos seis anos com humanos, foram encontrados cinquenta (50) estudos, depois realizada a leitura e analisados dos títulos e resumos chegou-se ao resultado final de três (3) estudos em relação a temática e problemática da pesquisa, os artigos encontravam-se no idioma inglês.

$\mathrm{Na}$ CINAHL o descritor Contexto (Co) não foi utilizado para se ter maior abrangência de estudo. Com a busca adaptada encontrou-se sessenta (60) estudos no total, sendo que limitando a busca para artigos com texto completo e realizado nos últimos seis anos com humanos, obteve-se quatro (4) estudos, sendo apenas dois (2) estudos foram condizentes com a questão desta pesquisa após a análise dos títulos e resumos, os artigos encontravam-se na língua inglesa.

Correspondendo à segunda fase da análise para a seleção dos artigos, eles foram avaliados quanto ao potencial de participação no estudo, observando o atendimento à questão de pesquisa, bem como o tipo de investigação, objetivos, amostra, método, desfechos, resultados e conclusão, resultando em um número final de treze (13) artigos.

$\mathrm{Na}$ análise e interpretação dos resultados foram analisadas as informações coletadas nos artigos científicos e criadas categorias analíticas que facilitou a ordenação e a sumarização de cada estudo. Essa categorização foi realizada de forma descritiva, indicando os dados mais relevantes para o estudo, possibilitando assim, uma compreensão imediata em relação aos mesmos.

A pesquisa levou em consideração os aspectos éticos da pesquisa quanto às citações dos estudos, respeitando a autoria das ideias, os conceitos e as definições presentes nos artigos incluídos na revisão.

Optou-se pela análise em forma estatística e de forma de texto, utilizando cálculos matemáticos e inferências, que estão apresentados em quadros e tabelas para facilitar a visualização e compreensão. As evidências científicas foram classificadas segundo os níveis e graus de recomendação propostos por Bork (2005).

\section{Resultados e Discussão}

Pode-se observar que os treze estudos incluídos nesta revisão, apresentavam abordagem quantitativa, correspondendo a $69,23 \%$ dos trabalhos; com delineamento transversal cerca de $92,31 \%$. O idioma predominante foi na língua portuguesa (61,54\%); com relação aos periódicos de publicações, o REUOL (15,38\%) foi o que mais forneceu estudos para esta amostra. Quanto à classificação de evidência 92,31\% eram nível seis. Sobre a localização dos estudos, o Brasil foi o país com maior frequência de estudos incluídos $(84,62 \%)$ (Tabela 1$)$. 
Tabela 1. Análise descritiva das produções cientificas acercar do processo de produção e compreensão do prontuário de enfermagem no ambiente hospitalar. Caxias, MA, Brasil, 2020. $(n=13)$.

\begin{tabular}{|c|c|c|}
\hline VARIAVEIS & $\mathbf{N}$ & $\%$ \\
\hline \multicolumn{3}{|l|}{ Base de dados } \\
\hline CINAHL & 2 & 15,38 \\
\hline BIREME & 8 & 61,54 \\
\hline PUBMED & 3 & 23,08 \\
\hline \multicolumn{3}{|l|}{ Abordagem do estudo } \\
\hline Quantitativo & 9 & 69,23 \\
\hline Qualitativo & 4 & 30,77 \\
\hline \multicolumn{3}{|l|}{ Ano } \\
\hline 2015 & 2 & 15,38 \\
\hline 2016 & 2 & 15,38 \\
\hline 2017 & 1 & 7,69 \\
\hline 2018 & 2 & 15,38 \\
\hline 2019 & 5 & 38,46 \\
\hline 2020 & 1 & 7,69 \\
\hline \multicolumn{3}{|l|}{ Idiomas } \\
\hline Inglês & 5 & 38,46 \\
\hline Português & 8 & 61,54 \\
\hline \multicolumn{3}{|l|}{ País } \\
\hline Brasil & 11 & 84,62 \\
\hline Roma & 1 & 7,69 \\
\hline EUA & 1 & 7,69 \\
\hline \multicolumn{3}{|l|}{ Delineamento de pesquisa } \\
\hline Estudo de caso & 1 & 7,69 \\
\hline Estudo Transversal & 12 & 92,31 \\
\hline \multicolumn{3}{|l|}{ Classificação da evidência } \\
\hline Cinco & 1 & 7,69 \\
\hline Seis & 12 & 92,31 \\
\hline \multicolumn{3}{|l|}{ Periódicos } \\
\hline Revista Nursing & 1 & 7,69 \\
\hline Elsevier & 1 & 7,69 \\
\hline REUOL & 2 & 15,38 \\
\hline ACTA Paulista de Enfermagem & 1 & 7,69 \\
\hline $\begin{array}{l}\text { Rev. online de pesquisa: cuidado é } \\
\text { fundamental }\end{array}$ & 1 & 7,69 \\
\hline RECOM & 1 & 7,69 \\
\hline OBJN & 1 & 7,69 \\
\hline REBEn & 1 & 7,69 \\
\hline JPMH & 1 & 7,69 \\
\hline REPEn & 1 & 7,69 \\
\hline Rev.esc.enferm.USP & 1 & 7,69 \\
\hline Revista RENE & 1 & 7,69 \\
\hline
\end{tabular}

Legenda: N=número; \%=percentual. Fonte: Pesquisa direta (2020).

Os artigos incluídos nesta revisão foram devidamente referenciados com o nome dos autores, ano de publicação em ordem decrescente, o título do artigo; objetivo principal; perfil amostral e principais resultados. Os estudos foram identificados com a letra "A" seguida do número do trabalho, no qual variaram de A1 a A13. Conforme apresentados no Quadro 3.

Os trezes estudos fizeram caracterização sobre a importância dos conteúdos registrados pela equipe multidisciplinar. A percepção dos profissionais multidisciplinares em seus respectivas erros, relataram a importância da informatização direta e a necessidade de fundamentos legais do prontuário (yeleye, 2019; Ferreira et al., 2020; Alves et al., 2018; Silva, Dias, \& Leite, 2019; Dutra et al., 2016; Azzolini et al., 2019; Candido et al., 2018). No que se refere a estrutura e composição do prontuário, evidenciou-se que a própria equipe dificulta seus registros gerando falhas e aberturas para novos erros (Alves et al., 
2018; Caldeira et al., 2019; Oyeleye, 2019; Meneses, Bezerra, Trajano \& Soares, 2015; Borges, Azevedo, Amorim, Figueiredo, \& Ribeiro, 2017; Diniz, Silva, Figueiredo, \& Tonini 2015).

Quanto ao uso de nomenclaturas indiscriminada, apontaram que, cerca de 35,8\% não eram padronizadas pelo serviço hospitalar, e algumas abreviações impossibilitaram a identificação dos procedimentos (Carneiro, Dutra, Costa, Mendes, \& Arreguy-Sena, 2016; Silva et al., 2019; Diniz et al., 2015; Ferreira et al., 2020). Em relação a infrações dos registros médicos, evidenciou-se falhas na visita clínica, déficit verbal, erros como não documentar eventos clínicos que ocorreram e documentar falsamente eventos que não ocorreram (Azzolini et al., 2019; Oyeleye, 2019; Meneses et al., 2015).

Alguns estudos revelaram que a sobrecarga de atividades, insuficiência de profissionais, a falta de tempo e baixa remuneração, leva a uma má qualidade do cuidado, ocasionando erros nos dados coletados, contextualizados e repassado (Silva et al., 2019; Borges et al., 2017; Meneses et al., 2015; Azevedo, Guedes, Araújo, Maia, \& Cruz 2019; Diniz et al., 2015; Candido et al., 2018; Alves et al., 2018). As principais causas de erros na prática do enfermeiro, com alta complexidade de risco no tratamento terapêutico do cliente está relacionado à ausência de priorização do paciente e a não abordagem da sistematização da assistência de enfermagem (Caldeira et al., 2019; Candido et al., 2018; Carneiro et al., 2016; Silva et al., 2019; Meneses et al., 2015). 


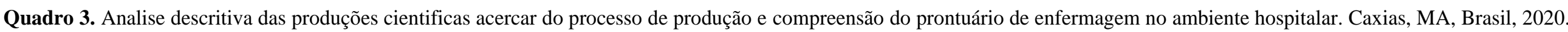
$(n=13)$.

\begin{tabular}{|c|c|c|c|c|c|}
\hline $\begin{array}{l}\mathrm{N}^{\circ} \text { de ordem } \\
\text { e base }\end{array}$ & Título do artigo & Autor/ ano & $\begin{array}{l}\text { Delineamento } \\
\text { do estudo }\end{array}$ & Objetivo principal & Perfil amostral \\
\hline $\begin{array}{c}\text { BIREME } \\
\text { A1 }\end{array}$ & $\begin{array}{l}\text { Uso de abreviaturas nos } \\
\text { registros de enfermagem em } \\
\text { um hospital de ensino. }\end{array}$ & $\begin{array}{l}\text { Carneiro et } \\
\text { al. }(2016)\end{array}$ & $\begin{array}{c}\text { Estudo } \\
\text { Transversal }\end{array}$ & $\begin{array}{l}\text { Avaliar a utilização de abreviaturas nos } \\
\text { registros de enfermagem de um hospital de } \\
\text { ensino descrevendo o perfil nos diferentes } \\
\text { setores, turnos de trabalho e categorias } \\
\text { profissionais da equipe de enfermagem. }\end{array}$ & $\begin{array}{l}\text { Foram coletados } 627 \text { registros de prontuários } \\
\text { arquivados no Serviço de Arquivo Médico e } \\
\text { Estatística do hospital, no período de março a } \\
\text { maio de } 2013 \text {. }\end{array}$ \\
\hline $\begin{array}{l}\text { BIREME } \\
\text { A2 }\end{array}$ & $\begin{array}{l}\text { Análise dos registros de } \\
\text { técnicos de enfermagem e } \\
\text { enfermeiros em prontuários. }\end{array}$ & $\begin{array}{l}\text { Ferreira et } \\
\text { al. }(2020)\end{array}$ & $\begin{array}{c}\text { Estudo } \\
\text { Transversal }\end{array}$ & $\begin{array}{l}\text { Analisar as principais não conformidades dos } \\
\text { registros de enfermagem de um hospital } \\
\text { público do Nordeste na cidade de Natal. }\end{array}$ & $\begin{array}{l}\text { Analisou-se nas enfermarias de clínica médica } \\
\text { e cirúrgica os registros de enfermagem dos } 10 \\
\text { primeiros dias de internação em } 120 \\
\text { prontuários selecionados. }\end{array}$ \\
\hline $\begin{array}{c}\text { BIREME } \\
\text { A3 }\end{array}$ & $\begin{array}{l}\text { Anotações da equipe de } \\
\text { enfermagem: } \\
\text { (des)valorização do cuidado } \\
\text { pelas informações fornecidas. }\end{array}$ & $\begin{array}{l}\text { Caldeira et } \\
\text { al. (2019) }\end{array}$ & $\begin{array}{c}\text { Estudo } \\
\text { Transversal }\end{array}$ & $\begin{array}{l}\text { Analisar as anotações da equipe de } \\
\text { enfermagem nos prontuários de crianças } \\
\text { hospitalizadas e discutir as informações } \\
\text { descritas pela enfermagem e suas implicações } \\
\text { para a assistência à criança. }\end{array}$ & $\begin{array}{l}\text { Os dados foram constituídos por anotações de } \\
\text { enfermagem em } 26 \text { prontuários de crianças } \\
\text { que estavam hospitalizadas no mês de janeiro } \\
\text { de 2014. Foram utilizados todos os } \\
\text { prontuários disponíveis no período de coleta } \\
\text { de dados. }\end{array}$ \\
\hline $\begin{array}{l}\text { BIREME } \\
\text { A4 }\end{array}$ & $\begin{array}{l}\text { A elaboração de evolução de } \\
\text { enfermagem e possíveis } \\
\text { dificuldades: percepção do } \\
\text { enfermeiro. }\end{array}$ & $\begin{array}{l}\text { Silva et al. } \\
\text { (2019) }\end{array}$ & $\begin{array}{c}\text { Estudo } \\
\text { Transversal }\end{array}$ & $\begin{array}{l}\text { Analisar percepção dos enfermeiros de um } \\
\text { hospital público do município de Belém, } \\
\text { acerca de possíveis dificuldades, para } \\
\text { elaboração de evoluções escritas. }\end{array}$ & $\begin{array}{l}\text { Os dados foram coletados no mês de setembro } \\
\text { de } 2016 \text {. Foram participantes do estudo oito } \\
\text { enfermeiros, que atuavam nas referências } \\
\text { clinicas, nos três turnos, cada entrevista foi } \\
\text { realizada com gravações das falas transcritas } \\
\text { na integra. }\end{array}$ \\
\hline $\begin{array}{l}\text { BIREME } \\
\text { A5 }\end{array}$ & $\begin{array}{l}\text { Importância das anotações de } \\
\text { enfermagem segundo a equipe } \\
\text { de enfermagem: implicações } \\
\text { profissionais e institucionais. }\end{array}$ & $\begin{array}{l}\text { Borges et al. } \\
\quad(2017)\end{array}$ & $\begin{array}{l}\text { Estudo de } \\
\text { Caso }\end{array}$ & $\begin{array}{l}\text { Compreender a importância das anotações de } \\
\text { enfermagem no prontuário do paciente para a } \\
\text { equipe de enfermagem; analisar as } \\
\text { implicações profissionais e institucionais dos } \\
\text { registros de enfermagem. }\end{array}$ & $\begin{array}{l}\text { Foram participantes } 12 \text { membros da equipe de } \\
\text { enfermagem lotados no cenário de pesquisa } \\
\text { compreendido como uma enfermaria clínico } \\
\text { cirúrgica de hospital de ensino de Minas } \\
\text { Gerais. }\end{array}$ \\
\hline $\begin{array}{l}\text { BIREME } \\
\text { A6 }\end{array}$ & $\begin{array}{l}\text { Prontuário do paciente: } \\
\text { qualidade dos registros na } \\
\text { perspectiva da equipe } \\
\text { multiprofissional. }\end{array}$ & $\begin{array}{l}\text { Meneses et } \\
\text { al. (2015) }\end{array}$ & $\begin{array}{c}\text { Estudo } \\
\text { Transversal }\end{array}$ & $\begin{array}{l}\text { Identificar a qualidade dos registros de } \\
\text { enfermagem na perspectiva da comunicação } \\
\text { multiprofissional; } \\
\text { Investigar as normas e regulamentos sobre o } \\
\text { registro no prontuário do paciente que } \\
\text { permitam a implantação do Processo de } \\
\text { Enfermagem. }\end{array}$ & $\begin{array}{l}\text { Foram analisados } 24 \text { prontuários de pacientes } \\
\text { internados, respeitando os requisitos } \\
\text { previamente definidos, são eles: paciente } \\
\text { internado a mais de uma semana, assistido por } \\
\text { pelo menos } 03 \text { profissionais. }\end{array}$ \\
\hline
\end{tabular}




\begin{tabular}{|c|c|c|c|c|c|}
\hline $\begin{array}{l}\text { BIREME } \\
\text { A7 }\end{array}$ & $\begin{array}{l}\text { Identificação do paciente nos } \\
\text { registros dos profissionais de } \\
\text { saúde. }\end{array}$ & $\begin{array}{l}\text { Alves et al. } \\
\text { (2018) }\end{array}$ & $\begin{array}{c}\text { Estudo } \\
\text { Transversal }\end{array}$ & $\begin{array}{l}\text { Identificar a conformidade dos dados de } \\
\text { identificação do paciente nos registros dos } \\
\text { profissionais de saúde de três hospitais } \\
\text { públicos do Rio Grande do Norte. }\end{array}$ & $\begin{array}{l}\text { Foram avaliados } 234 \text { prontuários. A coleta de } \\
\text { dados ocorreu mediante um instrumento } \\
\text { construído a partir de um Scoping review. }\end{array}$ \\
\hline $\begin{array}{l}\text { BIREME } \\
\text { A8 }\end{array}$ & $\begin{array}{llr}\text { Informações } & \text { de } & \text { Enfermagem } \\
\text { registradas } & \text { nos prontuários } \\
\text { frente às } & \text { exigências } & \text { do } \\
\text { Conselho } & \text { Federal } & \text { de } \\
\text { Enfermagem. } & & \end{array}$ & $\begin{array}{l}\text { Candido et } \\
\text { al. (2018) }\end{array}$ & $\begin{array}{c}\text { Estudo } \\
\text { Transversal }\end{array}$ & $\begin{array}{l}\text { Avaliar o padrão de registros de Enfermagem } \\
\text { em relação aos requisitos estabelecidos pela } \\
\text { instituição campo de estudo e Resolução } \\
\text { COFEN-272/2002 quanto à Sistematização da } \\
\text { Assistência de Enfermagem (SAE) }\end{array}$ & $\begin{array}{l}\text { Foram analisados } 287 \text { prontuários dos setores: } \\
\text { clínica médica, clínica cirúrgica, unidade de } \\
\text { terapia intensiva adulto (UTI) e pediátrico } \\
\text { (UTIP), unidade centro-cirúrgico (UCC), } \\
\text { unidade cirúrgica ambulatorial } \\
\text { (UCA) } \\
\text { pediatria. }\end{array}$ \\
\hline $\begin{array}{c}\text { CINAHL } \\
\text { A9 }\end{array}$ & $\begin{array}{l}\text { Examining } \text { Board Discipline } \\
\text { of Nurse Practitioners for } \\
\text { Medical Records Infractions. }\end{array}$ & $\begin{array}{l}\text { Oyeleye } \\
(2019)\end{array}$ & $\begin{array}{c}\text { Estudo } \\
\text { Transversal }\end{array}$ & $\begin{array}{l}\text { Examinar as práticas de prontuários médicos } \\
\text { dos PNs que foram disciplinadas pelo } \\
\text { Conselho de Enfermagem do Texas, com o } \\
\text { objetivo de esclarecer os PNs sobre tais } \\
\text { práticas e, idealmente, preveni-las. }\end{array}$ & $\begin{array}{l}\text { Os registros revisados neste estudo foram os } \\
\text { publicados entre abril de } 2015 \text { e abril de } 2018 \text {. }\end{array}$ \\
\hline $\begin{array}{l}\text { CINAHL } \\
\text { A10 }\end{array}$ & $\begin{array}{l}\text { Quality of nursing records: } \\
\text { analytical reflections on its } \\
\text { forms and contentes. }\end{array}$ & $\begin{array}{l}\text { Diniz et al. } \\
\text { (2015) }\end{array}$ & $\begin{array}{c}\text { Estudo } \\
\text { Transversal }\end{array}$ & $\begin{array}{l}\text { Analisar a qualidade dos registros efetuados } \\
\text { pela equipe de enfermagem. }\end{array}$ & $\begin{array}{l}\text { Foram analisados } 220 \text { registros de enfermeiros } \\
\text { e } 406 \text { registros de técnicos/auxiliares de } \\
\text { enfermagem, com um total de } 626 \text { registros. }\end{array}$ \\
\hline $\begin{array}{l}\text { PUBMED } \\
\text { A11 }\end{array}$ & $\begin{array}{l}\text { Nursing records at a teaching } \\
\text { hospital: a quasi-experimental } \\
\text { study. }\end{array}$ & $\begin{array}{l}\text { Dutra et al. } \\
\quad(2016\end{array}$ & $\begin{array}{c}\text { Estudo } \\
\text { Transversal }\end{array}$ & $\begin{array}{l}\text { Avaliar os registros da equipe de enfermagem } \\
\text { quanto ao cumprimento da legislação } \\
\text { específica de um hospital universitário, } \\
\text { considerando o setor da instituição, turno de } \\
\text { trabalho, categoria profissional e número de } \\
\text { ofensas por registro antes e após a intervenção } \\
\text { educativa. }\end{array}$ & $\begin{array}{l}\text { Foi realizada uma avaliação preliminar e outra } \\
\text { avaliação dos registros de enfermagem da } \\
\text { instituição após a conclusão do experimento } \\
\text { (atividade educativa). }\end{array}$ \\
\hline $\begin{array}{l}\text { PUBMED } \\
\text { A12 }\end{array}$ & $\begin{array}{lrr}\text { Quality } & \text { improvement of } \\
\text { medical records through } \\
\text { internal auditing: } \\
\text { comparative analysis. }\end{array}$ & $\begin{array}{l}\text { Azzolini et } \\
\text { al. (2019) }\end{array}$ & $\begin{array}{c}\text { Estudo } \\
\text { Transversal }\end{array}$ & $\begin{array}{l}\text { Avaliar as diferenças de qualidade dos } \\
\text { registros médicos antes e depois da auditoria } \\
\text { interna em um hospital de ensino de terceiro } \\
\text { nível italiano. }\end{array}$ & $\begin{array}{l}\text { Foi feita uma seleção proporcional de } \\
\text { prontuários com base no número de admissões } \\
\text { do ano anterior para cada ala. }\end{array}$ \\
\hline $\begin{array}{l}\text { PUBMED } \\
\text { A13 }\end{array}$ & $\begin{array}{l}\text { Documentation of the nursing } \\
\text { process in public health } \\
\text { institutions. }\end{array}$ & $\begin{array}{l}\text { Azevedo et } \\
\text { al. (2019) }\end{array}$ & $\begin{array}{c}\text { Estudo } \\
\text { Transversal }\end{array}$ & $\begin{array}{l}\text { Identificar a prevalência de documentação do } \\
\text { PE nos hospitais e ambulatórios administrados } \\
\text { pela SES/SP. }\end{array}$ & $\begin{array}{l}\text { Foram entrevistas presenciais com a } \\
\text { enfermeira responsável pelo setor. Todos os } \\
\text { setores de atendimento ( } 429) \text { e } 28 \text { setores } \\
\text { entre diferentes instituições. }\end{array}$ \\
\hline
\end{tabular}

Fonte: Artigos Pesquisados (2020). 
Os trezes estudos tiveram como enfoque principal os aspectos do prontuário e as ações englobando o núcleo da instituição hospitalar. Quanto as frequências de conformidades e não conformidades, nenhum dos indicadores auditados atingiu 100\% de conformidade nas anotações do executor (Ferreira et al., 2020; Alves et al., 2018; Azevedo et al., 2019; Candido et al., 2018; Diniz et al., 2015; Dutra et al., 2016; Borges et al., 2017; Azzolini et al., 2019). Com relação às características dos registros de enfermagem, estudos citaram que o prontuário deve fornecer dados inerentes e indispensáveis ao processo de cuidar (Meneses et al., 2015; Caldeira et al., 2019; Borges et al., 2017; Candido et al., 2018; Ferreira et al., 2020; Diniz et al., 2015; Oyeleye, 2019; Dutra et al., 2016).

Em relação à identificação da categoria profissional, reportaram que os enfermeiros, médicos, técnicos e auxiliares de enfermagem foram os mais predominantes (Dutra et al., 2016; Azzolini et al., 2019; Ferreira et al., 2020; Carneiro et al., 2016; Caldeira et al., 2019; Diniz et al., 2015; Candido et al., 2018; Borges et al., 2017; Alves et al., 2018; Silva et al., 2019; Meneses et al., 2015; Azevedo et al., 2019; Oyeleye, 2019). Quanto a fase da assistência, foi citado que os cuidadores precisam ter conhecimentos e prática dos respectivos itens que correspondem ao prontuário (Silva et al., 2019; Ferreira et al., 2020; Meneses et al., 2015; Azevedo et al., 2019; Oyeleye, 2019; Caldeira et al., 2019; Borges et al., 2017; Candido et al., 2018).

As evidências incluídas nesta revisão destacaram ainda a presença de acometimentos, desordens na estruturação do prontuário e falhas no diálogo, reportado exclusivamente nos setores do âmbito hospitalar (Oyeleye, 2019; Dutra et al., 2016; Candido et al., 2018; Carneiro et al., 2016; Borges et al., 2017; Azevedo et al., 2019).

O ambiente hospitalar é constituído por diferentes indicadores externos e internos, que colaboram no processo do trabalho, desde a sua visibilidade de organização até as orientações fornecidas e utilizadas (Meneses et al., 2015; Caldeira et al., 2019; Dutra et al., 2016; Azzolini et al., 2019; Carneiro et al., 2016; Alves et al., 2018; Silva et al., 2019; Azevedo et al., 2019; Borges et al., 2017).

A apresentação da discussão está organizada em duas sessões para melhor análises e compreensão das informações, com base aos objetivos que compuseram esta revisão integrativa, abrangendo: a caracterização do prontuário frente às categorias, setor e fase da assistência, e os Indicadores de conformidades e não conformidades e seu impacto para o paciente hospitalizado.

\section{Caracterização do prontuário frente às categorias, setor e fase da assistência}

Nos achados relacionados aos setores que tiveram incidência notadamente maior de danos, destacam-se as áreas clínica médica, obstétrica e internação geral, os quais relatam que uma má qualidade de assistência resulta no aumento de patologia e novos agravantes referente à saúde do cliente. Infere-se que esses achados sejam devidos a ausência de competência, tornando-se essencial à instituição, junto com sua gestão hospitalar, orientar e desenvolver estratégias de qualificação de serviço e informar as normas que cada setor deve seguir (Euzebio, Ceretta, \& Soratto, 2017; Silva et al., 2017; Camargo \& Pereira, 2017).

Dentre os fatores que levam ao não uso de abreviaturas e siglas padronizadas, Araujo et al. (2017) revelaram que as abreviaturas e siglas não padronizadas estão associadas nas modalidades dos setores, pela linguagem utilizada, a grafia idêntica, ou à similaridade ao determinado termo na documentação. Estudos similares mostram que os profissionais apresentam entendimento e interpretação equivocadas das anotações e fragilidades com ausência de informações antecipadas sobre a saúde do paciente, quando comparadas por diferentes setores (Silva et al., 2017; Carneiro et al., 2016; Santana \& Araújo, 2016)

Com relação aos fatores que levam ao real registros dos horários, os estudos de Hammes (2019), Barreto, Lima e Xavier (2016) e o de Camargo e Pereira (2017) citaram que o profissional da saúde deve sempre anotar o horário de saída do paciente e a forma que saiu, se saiu acompanhado ou não; registrar ainda o estado de saúde do paciente; registrar como foi 
realizada a alta médica, se foi administrativa ou a pedido do paciente, do membro da família, ou de outras pessoas envolvidas na responsabilidade do paciente.

As evidências corroboraram entre si ao mencionarem que a excessiva prestação de serviços pode-se tornar um problema que atinge a assistência ofertada ao paciente, facilitando assim, tendências progressivas de erros acometidos, ou seja, muitos profissionais da equipe de enfermagem atuam em um ou mais empregos por dia, desencadeando, dessa forma, sobrecarga de tarefas, desgaste, queda de produtividade e na organização, desmotivação e estresse (Santana \& Araújo, 2016; Silva et al., 2017; Euzebio et al., 2017).

Em sintonia com Trindade e Lopes (2019), constataram-se que os profissionais de saúde são a forma mais prática de verificação do trabalho prestado ao cliente; mas, quando a equipe de enfermagem deixa de identificar as anotações, e a partir do momento que não existem registros de tal procedimento, materiais e medicamentos efetuados, ao serem glosados pela auditoria hospitalar, podem culminar com um prejuízo financeiro para o serviço hospitalar, pois se não foi registrado, não foi realizado; portanto, não tem motivo para pagamento de algum serviço.

Quanto à identificação da categoria profissional, os estudos analisados enfatizaram que enfermeiros e técnicos de enfermagem realizam as anotações por turnos e não por hora cronológica, o que influencia no aparecimento de espaços em branco ao longo do impresso (Araujo et al., 2017; Santana \& Araújo, 2016). O que diverge em parte dos estudos de Barreto, et. (2016) e Dias, Faria, Fleury, Jorge e Nascimento (2018), nos quais pode-se constatar também que os profissionais não se identificavam corretamente, não utilizavam identificação por meio de carimbo, rubrica ou o número do COREN, ou seja, cada profissional cometia diferentes infrações específicas.

Frente aos resultados alcançados da fase da assistência, muitos estudos apontaram os tipos de fragilidades. Nos estudos de Araujo et al. (2017), Gomes, Cubas, Pleis, Shmeil e Peluci (2016) e Barreto et al. (2016), apresentaram como os mais frequentes os respectivos exames físicos, diagnósticos, tratamento, evoluções, transferência de unidade, alta e óbito. Complementando ainda que o profissional não deve omitir nenhuma informação favorecendo o seu reconhecimento pelos membros da equipe de saúde.

De acordo com Vaidotas et al. (2019) e Hammes (2019), os fatores mais citados inerentes às ações direcionadas ao médico, foi a sua conduta não profissional que muitas das vezes tinha fraudes pertinentes e assim acabava prejudicando no tratamento do paciente. Os estudos de Garritano, Junqueira, Lorosa, Fujimoto e Martins (2020) e Araújo, Rechmann e Magalhães (2019) conduziram a outros fatores específicos, como as inconsistências na implementação de medidas seguras e o não cumprimento das práticas avançadas; sendo, portanto, menos efetuado na avaliação clínica completa.

\section{Indicadores de conformidades e não conformidades e seu impacto para o paciente hospitalizado}

As evidências de Candido et al. (2018) revelaram que o padrão e conteúdo do prontuário encontram-se em uniformidade repetitiva se for comparada com o padrão e normas da instituição. Estudos precedentes de Borges (2016) e Hammes (2019), citaram que o preenchimento das fichas e checklists não é realizado corretamente, com informações insuficientes, colocando um risco potencial, já que não foram registrados os dados necessários na entrada do paciente.

Para minimizar as inconformidades e inadequações do prontuário de acordo com os estudos utilizados nessa revisão, é através do correto preenchimento dos itens relativos à identificação do paciente que isso se dá. Corroboraram com esses achados os estudos de Ciocca (2014), Coltri e Silva (2019) e o de Camargo e Pereira (2017), os quais recomendam aos enfermeiros atenderem à legislação vigente e os critérios legais para proceder uma anotação segura e completa; além disso, o dimensionamento correto do profissional identifica e previne erros, que muitas vezes, é solucionado na hora que são coletados os dados do paciente, sem mesmo precisar de uma avaliação ampla, recorrente as etapas dos procedimentos clínicos. 
Os achados relacionados à ilegibilidade no prontuário do cliente hospitalizado encontram-se principalmente na evolução de enfermagem e nas prescrições médicas. Para diminuição da interpretação equivocada e o desconhecimento da legibilidade, os estudos de Barreto et al. (2016) e Garritano et al. (2020) acrescentaram que o profissional, antes de executar tal ação, precisa compreender e ter conhecimento do que está escrito. Outro ponto é procurar o autor responsável pela anotação e pedir clareza das anotações e principalmente informar a melhoria da sua escrita.

No estudo de Candido et al. (2018), no que se refere às falhas que ocorreram na admissão, foram encontradas anotações indevidas, como omissão das queixas relacionadas a dor, procedimentos de operação cirúrgica, presenças de lesões prévias e mensuração dos sinais vitais. O que diverge dos estudos incluídos de Vaidotas et al. (2019), Borges (2016), Garritano et al. (2020) e Barboza (2018), os quais evidenciaram erros de trocas dos medicamentos, o uso excessivo de materiais, punção venosa e as vias de administração, sendo os itens mais atingidos de ocorrências ao tratamento.

Dentro os fatores que levam a adesão da enfermagem em relação a SAE, os estudos de Barreto et al. (2016) e o de Candido et al. (2018) revelaram que a adesão é satisfatória, quando realizada sem barreira; mas, ao avaliar as frequências de inconformidades das anotações anexadas no prontuário, conclui-se que no ambiente hospitalar faz-se necessário ter uma fiscalização do superior, desenvolver programas educacionais permanentes, para contribuir com subsídios na sistematização da assistência de enfermagem e assim garantir uma nova ferramenta que contribuirá para a segurança do cliente, assim como, na qualidade das respostas das ações realizadas.

Dentro da concepção de Santos (2019), revelou-se que no ambiente hospitalar é notado constantemente o uso de etiquetas de identificação incoerentes e incompletas. Em contrapartida, os estudos realizados por Trindade e Lopes (2019) e Silva et al. (2017), apontam que os diversos erros está associado na pulseira do cliente e da mãe, em que ocorre a troca do nome da criança, ou então, a ausência de alguma letra e a ausência do número do prontuário do paciente.

De acordo com os estudos analisados, o cuidador deve ter atenção no momento de realizar a administração de medicamentos. Os estudos de Garritano et al. (2020) enfatizaram que o profissional, em todos os casos, não deve esquecer de fazer as anotações ao lado em que o medicamento foi realizado, registrar o tipo de dispositivo utilizado, providências adotadas, se o paciente teve rejeição à dose, presença de alergias e observar o local se tem alguma interferência. Diante dos estudos de Araújo et al. (2019) e Hammes (2019), afirmam-se que, uma vez que, os atos errados são expostos ao paciente, maior é o quadro de complicações extremas que ele irá apresentar.

Os achados relacionados à ocorrência de erro de prescrição de medicações registrado no prontuário de enfermagem, de acordo com os estudos de Garritano et al. (2020) e Vaidotas et al. (2019), apontaram falhas em medicamento não autorizado, dose errada, medicação contraindicada ao paciente, ao anotar um evento ou atividade clínica que não ocorreu, ou então assinar uma ação errônea de outro cuidador, sem reconhecer o erro cometido pelo executor, e a reincidência de prescrições de dias anteriores sem analise.

Dentre os fatores que levam ao papel da auditoria em saúde, os estudos de Garcia, Ferreira e Silva (2018) revelaram que a auditoria surgiu com a finalidade de avaliar a qualidade da assistência; para tanto, somente será possível se o prontuário estiver com informações fidedignas da prestação do serviço. Corroborando com os resultados de Lima et al. (2018), Tiburcio, Sousa e Santos (2019) e Loureiro, Da Costa, Marques e Hoyashi (2018), relataram que, por meio da auditoria é possível desenvolver indicadores assistenciais que auxiliem na melhoria do cuidado ao paciente.

A auditoria em enfermagem é um método de barreira mais eficaz, de acordo com os estudos utilizados nessa revisão, tendo em vista que, fornecem ideias para os gestores, corroborando com as evidências de Santana e Araújo (2016) e Loureiro et al. (2018). De acordo com as evidências científicas analisadas, além de proporcionar a qualidade do trabalho, evita as glosas hospitalares, minimiza os prejuízos, processos judiciais, custos por cobrança, gastos desnecessários e consequentemente, contribuem com a organização hospitalar (Trindade \& Lopes, 2019; Euzebio et al., 2017; Santos, 2019). 
O estudo de Tiburcio et al. (2019) aponta-se que o enfermeiro auditor é um profissional habilitado e capacitado para lidar com as demandas do ambiente hospitalar, sendo uma função exercida com respaldo legal, autonomia e responsabilidade. Um enfermeiro auditor deve buscar atualizações na sua área promissora, e sempre esclarecer os fatos que podem alterar o objetivo principal dos registros, com a finalidade de impedir a elaboração e interpretação errônea para que não haja inconformidades, sem fundamentos.

De acordo com os estudos analisados, o prontuário eletrônico é uma ferramenta eficiente que prova a falta de inveracidade ou a inexatidão de informações. Corroboram com esses achados os estudos de Coltri e Silva (2019), Rago e Zucchi (2017) e Carvalho (2017). Estudo recente de Vaidotas et al. (2019) citou que o prontuário eletrônico consiste em apoio para uma melhor assistência, possibilitando mais organização, reduz a existência de erros, facilita a agilidade do registro e acesso aos dados, evitando assim, os desperdícios de papeis armazenados, no caso do prontuário analógico, em que sofre perdas de estrutura e conteúdo com tempo.

Quanto à presunção de veracidade das informações de um prontuário digitalizado, os estudos de Coltri e Silva (2019), afirmam que, se o sistema eletrônico não possuir a certificação dos dados, a presunção de veracidade das informações seguirá as mesmas regras e termos do prontuário informatizado em papel, através da atribuição específica da Comissão de Revisão de Prontuário.

De acordo com os achados analisados de Martins, Sartor e Silva (2019), estes defendem que os profissionais multidisciplinares carecem de um ensino avançado na nova tecnologia de informações, pois a grande maioria demonstra um elevado grau de insatisfação, afirmando que o dispositivo eletrônico é difícil de acessar, considerando um grande obstáculo, pois muitos não sabem digitar, e durante o atendimento eles ficam mais presos no dispositivo, causando lentidão na assistência, deixando de lado a presença real do paciente e, principalmente, inexistindo a interação entre profissional e paciente.

Frente a um prontuário devidamente organizado e estruturado de acordo com os padrões estabelecidos, os estudos de Araújo et al. (2019), Camargo e Pereira (2017) e Silva et al. (2017) reforçam que o binômio profissional de enfermagem/instituição de saúde permita-se reconhecer e corrigir os erros, buscar treinamentos com relação ao trabalho desenvolvido pelos cuidadores, e a inovação na área da saúde. Outras atribuições especificas seriam fortalecer o padrão de zelo, seu valor legal, e o direito do cliente e a família de terem acesso às informações do prontuário. Dessa forma, manter o prontuário completo e preciso não protege somente a rescisão do trabalho, mas principalmente a vida do cliente.

\section{Considerações Finais}

$\mathrm{O}$ estudo permitiu identificar e descrever a frequência de intercorrências obtidas no prontuário da equipe de enfermagem. Os resultados evidenciaram uma considerável prevalência de erros na evolução e no preenchimento da procedência do paciente, o uso de terminologias inapropriadas, e a não checagem adequada das prescrições, em que foram notórias as falhas de medicações realizadas, com a repetição de prescrições de dias anteriores, sem análise. Revelou-se que, o diálogo entre os profissionais não tinha ligação com o escrito, resultando em descontinuidade da assistência, e boa parte do acervo encontrava-se com perda de fichas, uso de corretivos, lacunas e rasuras, destacando assim, o desinteresse do cuidador.

Apresentam-se como limitações deste estudo, baixo nível de informações relevantes a respeito do prontuário de enfermagem, bem como o número inferior de artigos dentro do recorte temporal. Outras limitações foi adaptar a estratégia de busca, e o fato de não ter conteúdos em algumas bases de dados, em que foi possível observar que boa parte das publicações de interesse não estava disponível na integra para download, o que resultou em percalços para a elaboração dessa pesquisa.

No cenário atual, a enfermagem encontra-se inserida em diversas áreas ocupacionais; dessa maneira, cabe ao profissional adotar medidas de capacitação permanente para a prática assistencial, sempre em conjunto aos aspectos éticospolítico da profissão. Diante disso, deve-se buscar novas atualizações sustentadas em bases cientificas, pautando sempre a 
adesão correta do prontuário, e possibilitar a resolutividade sobre o seu serviço fragmentado. O que se atesta é aprender com seus próprios erros, e proporcionar a satisfação dos pacientes, familiares e do hospital.

Contudo, através desse estudo ficou evidente a premência de promover a excelência do prontuário analógico. Esperase que esta pesquisa possa ampliar as possibilidades de um melhor serviço de enfermagem, contribuir para a produtividade da instituição, e servir de investimento na área educativa. Sobretudo, almeja-se que os profissionais, desde a graduação, formação e a atuação, vislumbram a reais abrangências das necessidades da sua profissão, buscando conhecimento geral sobre o acervo informatizado, principalmente do que está anotando e onde está anotando, a fim de que não haja complicações nas ações a serem realizadas ao paciente.

\section{Referências}

Alves, K. Y. A., Oliveira, P. T. C., Chiavone, F. B. T., Barbosa, M. L., Saraiva, C. O. P. O., Martins, C. C. F., \& Santos, V. E. P. (2018). Identificação do paciente nos registros dos profissionais de saúde. Acta Paulista de Enfermagem, 31(1), 79-86.

Araújo, A. T. M., Rechmann, I. L., \& Magalhães, T. A. (2019). O sigilo do prontuário médico como um direito essencial do paciente: uma análise a partir das normativas do conselho federal de medicina. Cadernos Ibero-americanos de Direito Sanitário, 8(1), 95-109.

Araujo, M. M., Diniz, S. O. S., \& Silva, P. S. (2017). Registros de enfermagem: reflexões sobre o cotidiano do cuidar. Abcs Health Sciences, 42(3), 161-165.

Azevedo, O. A., Guedes, É. S., Araújo, S. A. N., Maia, M. M., \& Cruz, D. A. L. M. (2019). Documentação do processo de enfermagem em instituições públicas de saúde. Revista da Escola de Enfermagem da USP, 53, e03471.

Azzolini, E., Furia, G., Cambieri, A., Ricciardi, W., Volpe, M., \& Poscia, A. (2019). Quality improvement of medical records through internal auditing: a comparative analysis. J Prev Med Hyg, 60, 250-255.

Barboza, V. S. (2018). A gestão documental na tramitação do prontuário do paciente na unidade de prontuário e estatística: observação e análise do arquivo médico do Hospital Universitário de Sergipe HU/UFS (Trabalho de Conclusão de Curso). Universidade Federal de Sergipe, Sergipe, São Cristóvão, SE, Brasil.

Barreto, J. A., Lima, G. G., \& Xavier, C. F. (2016). Inconsistências das anotações de enfermagem no processo de auditoria. Revista de Enfermagem do Centrooeste Mineiro, 6(1), 2082-2093.

Borges, F. F. D., Azevedo, C. T., Amorim, T. V., Figueiredo, M. A. G., \& Ribeiro, R. G. M. (2017). Importância das anotações de enfermagem segundo a equipe de enfermagem: implicações profissionais e institucionais. Revista de Enfermagem do Centro-oeste Mineiro, 7 , e1147.

Borges, M. S. (2016). O prontuário como sistema de informação em saúde: fatores que interferem na qualidade do preenchimento (Trabalho de Conclusão de Curso). Universidade Federal Fluminense, Escola de Enfermagem Aurora de Afonso Costa, Niterói, RJ, Brasil.

Bork, A. M. T. (2005). Enfermagem baseada em evidencias: Guanabara Koonga.

Caldeira, M. M., Souza, T. V., Morais, R. C. M., Moraes, J. R. M. M., Nascimento, L. C. N., \& Oliveira, I. C. S. (2019). Anotações da equipe de enfermagem: a (des)valorização do cuidado pelas informações fornecidas. J. res.: fundam. care. Online, 11(1), 135-141.

Camargo, L. R. L., \& Pereira, G. R. (2017). Análise dos registros realizados pela enfermagem e o possível impacto na auditoria: uma revisão da literatura nacional. Revista de Administração em Saúde, 17(68), 1-11.

Candido, A. S. G., Cunha, I. C. K. O., \& Munhoz, S. (2018). Informações de Enfermagem registradas nos prontuários frente às exigências do Conselho Federal de Enfermagem. Rev Paul Enferm, 29(1), 31-8.

Carneiro, S. M., Dutra, H. S., Costa, F. M., Mendes, S. E., \& Arreguy-Sena, C. (2016). Use of abbreviations in the nursing records of a teaching hospital. Revista da Rede de Enfermagem do Nordeste, 17(2), 208-16.

Carvalho, R. C. (2017). Aplicação de técnicas de mineração de texto na recuperação de informação clínica em prontuário eletrônico do paciente (Dissertação de mestrado). Faculdade de Filosofia e Ciências, Universidade Estadual Paulista, Marília, SP, Brasil.

Ciocca, I. G. (2014). O prontuário do paciente na perspectiva arquivística (Dissertação de mestrado) - Faculdade de Filosofia e Ciências, Universidade Estadual Paulista, Marília, SP, Brasil.

Coltri, M. V., \& Silva, R. H. A. (2019). Prontuário do paciente: comentários à lei nº 13.787/2018. Revista Brasileira de Odontologia Legal, 6(2), 89-105.

Dias, E. G., Faria, M. L. S., Fleury, A. T. S., Jorge, I. L., \& Nascimento, A. A. (2018). Análise das anotações de enfermagem de um hospital público. Revista Diálogos \& Ciência $(D \& C), 1,(41)$ : 11-21.

Diniz, S. O. S., Silva, P. S., Figueiredo, N. M. A., \& Tonini, T. (2015). Qualidade dos registros de enfermagem: reflexões analíticas em suas formas e conteúdos. Rev enferm UFPE on line, 9(10), 9616-23.

Dutra, H. S., Mendes, S. E., Carneiro, S. M., Costa, F. M., Barboza, R. C. P., \& Ribeiro, L. C. (2016). Nursing records at a teaching hospital: a quasiexperimental study. Online brazj nurs, 15(3), 351-360. 
Euzebio, A. M., Ceretta, L. B., \& Soratto, M. T. (2017). Avaliação dos registros de enfermagem em prontuários de pacientes internados em clínica cirúrgica. RIES, 6, n. (1), 188-199.

Ferreira, L. L., Chiavone, F. B. T., Bezerril, M. S., Alves, K. Y. A., Salvador, P. T. C. O., \& Santos, V. E. P. (2020). Analysis of records by nursing technicians and nurses in medical records. Revista Brasileira de Enfermagem, 73(2), e20180542.

Garcia, T. T., Ferreira, W. F. S., \& Silva, A. (2018). Processo de auditoria em enfermagem e suas dimensões na assistência ao paciente: uma revisão sistemática de literatura. Revista Eletrônica da FAINOR, 11(1), 1-30.

Garritano, C. R. O., Junqueira, F. H., Lorosa, E. F. S., Fujimoto, M. S., \& Martins, W. H. A. (2020). Avaliação do Prontuário Médico de um Hospital Universitário. Revista Brasileira de Educação Médica, 44(1), e009.

Gomes, D. C., Cubas, M. R., Pleis, L. E., Shmeil, M. A. H., \& Peluci, A. P. V. D. (2016). Termos utilizados por enfermeiros em registros de evolução do paciente. Revista Gaúcha de Enfermagem, 37(1), e53927.

Hammes, A. H. (2019). Avaliação do preenchimento de prontuários médicos em um hospital geral (Trabalho de Conclusão de Curso). Universidade Federal da Fronteira Sul, Passo Fundo, RS, Brasil.

Lima, J. R., Pimenta, C. J. L., Frazão, M. C. L. O., Da Silva, C. R. R., Viana, L. R. C., \& Salviano, G. R. (2018). Auditoria de enfermagem: revisão integrativa da literatura. Revista Nursing, 21(247), 2531-2534.

Loureiro, L. H., Da Costa, L. M., Marques, V. L., \& Hoyashi, C. M. T. (2018). Como a auditoria de enfermagem pode influenciar na qualidade assistencial. Revista Práxis, 9(2), 91-102.

Martins, L., Sartor, G. D., \& Silva, M. P. (2019). Prontuário Eletrônico do Paciente: Adoção de novas tecnologias de acesso. J. Health Inform., $11(3), 67-73$.

Meneses, L. B. A., Bezerra, A. F., Trajano, F. M. P., \& Soares, M. J. G. O. (2015). Prontuário do paciente: qualidade dos registros na perspectiva da equipe multiprofissional. Rev enferm UFPE on line, Recife, 9(10), 9485-91.

Oliveira, F. B. M., Costa, A. C. A. L., Alves, D. L., França, J. F., Macedo, M. S., \& Santos, R. D. (2016). Relação entre a sobrecarga de trabalho e erros de administração de medicação na assistência hospitalar. Revista Ciências \& Saberes, 2(2), 325-334.

Oyeleye, O. A. (2019). Examining Board Discipline of Nurse Practitioners for Medical Records Infractions. The Journal For Nurse Practitioners, 15(9), 613617.

Rago, C. A. P., \& Zucchi, P. (2017). Prontuário Eletrônico do Paciente: como a teoria da difusão de inovações pode colaborar na sua implantação. J Health Inform., 9(2), 57-61.

Santana, L. C., \& Araújo, T. C. (2016). Análise da qualidade dos registros de enfermagem em prontuários. Revista Acreditação: ACRED, 6(11), 59-71.

Santos, L. L. (2019). Auditoria de enfermagem e os registros de enfermagem: uma revisão de literatura (Trabalho de Conclusão de Curso). Faculdade de Ciências da Educação e Saúde, Centro Universitário de Brasília, Brasília, DF, Brasil.

Silva, A. G. I., Dias, B. R. L., \& Leite, M. R. (2019). A elaboração de evoluções de enfermagem e possíveis dificuldades: percepção do enfermeiro. Revista Nursing, 22 (254), 3039-42.

Silva, L. C. S., Prado, M. A., Barbosa, M. A., Ribeiro, D. P. O., L ima, F. H. A., Andrade, L. Z., \& Silva, A. P. B. (2017). Inconformidades acerca dos registros em prontuários: percepção dos trabalhadores de saúde da região central do Brasil. Anais do Congresso Ibero-Americano de Investigação Qualitativa, Lisboa, Portugal, 6 .

Tiburcio, A. P. N., Sousa, L. A. A., \& Santos, R. F. (2019). A importância do enfermeiro auditor nas instituições hospitalares. Psicologia e Saúde em Debate, $5(1), 50-59,15$.

Trindade, B. M. S., \& Lopes, A. (2019). Importância das anotações de enfermagem no processo de auditoria. Revista InterSaúde, 1(1), 47-58.

Vaidotas, M., Yokota, P. K. O., Negrini, N. M. M., Leiderman, D. B. D., Souza, V. P., Santos, O. F. P., \& Wolosker, N. (2019). Medication errors in emergency departments: is electronic medical record an effective barrier? Einstein, 17(4), eGS4282. 